\title{
RADIO Y EMPODERAMIENTO SOCIAL. USOS Y GRATIFICACIONES DEL TALLER DE RADIO PARA LAS PERSONAS CON DISCAPACIDAD INTELECTUAL O DEL DESARROLLO
}

\author{
Radio and social empowerment. Uses and the \\ rewards gained of radio workshops for individuals \\ with intellectual or developmental disabilities
}

Juan-Antonio Cortés-Fuentes y Beatriz Correyero-Ruiz

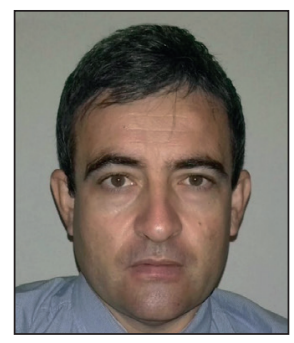

Juan-Antonio Cortés-Fuentes es doctor en periodismo por la Universidad Católica de Murcia (UCAM) y master en Dirección de Comunicación. Trabaja como periodista en Dipalme Radio, servicio público de radio de la Diputación de Almería. Ha trabajado y colaborado en medios de comunicación como Antena 3 Radio, La voz de Almería, Diario ideal, La crónica, y Radio televisión información $(R T I)$.

http://orcid.org/0000-0003-2346-7626

Dipalme Radio. Servicio público de radio de la Diputación de Almería C/ Navarro Rodrigo, 17. 04001 Almería, España jacortes@dipalme.org

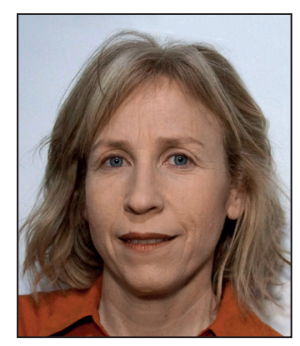

Beatriz Correyero-Ruiz es doctora en periodismo por la Universidad Complutense de Madrid y vicedecana del Grado en Periodismo de la Universidad Católica de Murcia (UCAM) donde imparte las asignaturas de Información en radio, Taller de periodismo multimedia y Practicum. Es miembro del grupo de investigación Digitalac y ha participado en varios proyectos de I+D financiados a nivel nacional.

http://orcid.org/0000-0003-0069-8448

Universidad Católica de Murcia Facultad de Ciencias Sociales y de la Comunicación, Departamento de Periodismo Campus de los Jerónimos, s/n. 30107 Guadalupe (Murcia), España bcorreyero@ucam.edu

\section{Resumen}

Se analiza la influencia que puede tener en una persona con discapacidad intelectual o del desarrollo la participación en talleres de radio (empoderamiento ciudadano -desarrollo de habilidades comunicativas y sociales, autoestima-; visibilidad social; comprensión de la discapacidad y alfabetización mediática). El estudio, basado en cuestionarios estructurados enviados a tutores y a sujetos participantes, presenta una imagen del funcionamiento de un taller de radio, confirma el aumento exponencial de los proyectos radiofónicos en los últimos cinco años al calor de las nuevas tecnologías de la información y la comunicación y revela un nuevo escenario de oportunidades de acceso al medio radio a través del ecosistema de Internet (radio online y alojamientos de podcasting). La investigación da a conocer el elevado grado de satisfacción que muestran las personas con discapacidad intelectual que colaboran en estos talleres.

\section{Palabras clave}

Radio; Talleres de radio; Radio online; Podcast; Discapacidad intelectual; Feaps; Alfabetización mediática; Visibilidad social; Empoderamiento ciudadano.

\begin{abstract}
We analyze the influence that participation in radio workshops (citizen empowerment -development of communicative and social abilities, self-esteem-, social visibility, understanding disability, and media literacy) has on individuals with intellectual or developmental disabilities. This study, which is based on structured questionnaires sent to tutors and participating subjects, presents a picture of the current workings of a radio workshop, confirms the exponential growth of radio projects in the last five years in light of the new technologies of information and communication, and reveals a new scenario of opportunities for accessing the radio medium through the Internet ecosystem (online radio and podcast hosting). The high degree of satisfaction shown by individuals with intellectual disabilities who collaborate in these workshops is revealed.
\end{abstract}




\section{Keywords}

Radio; Radio workshops; Online radio; Podcasts; Intellectual disability; Feaps; Media literacy; Social visibility; Citizen empowerment.

Cortés-Fuentes, Juan-Antonio; Correyero-Ruiz, Beatriz (2017). "Radio y empoderamiento social. Usos y gratificaciones del taller de radio para las personas con discapacidad intelectual o del desarrollo". El profesional de la información, v. 26, n. 1 , pp. 55-65.

https://doi.org/10.3145/epi.2017.ene.06

\section{Introducción}

En torno a 4 millones de españoles tienen algún tipo de discapacidad, según la Encuesta de discapacidad, autonomía personal y situaciones de dependencia (INE, 2008). Sin embargo, el tratamiento mediático que reciben no guarda proporción con la estadística poblacional que representan. Además, la utilización del lenguaje para referirse a este colectivo, aunque ha experimentado un avance cualitativo en la última década, sigue alimentando una línea discursiva que encierra el uso injustificado de adjetivos sustantivados como "discapacitado" o "minusválido" (Perujo-Serrano, 2002) y las informaciones que suelen aparecer en los medios se caracterizan por el tono victimista o caritativo (Madrigal-Barrón; Carrillo-Pascual 2013), no dan preeminencia a la persona sobre la discapacidad y están desprovistas de análisis contextual y causal (Casado, 2001).

El contexto mediático actual se caracteriza por la ausencia de alternativas reales de acceso a las plataformas tradicionales de comunicación de masas -televisión, radio y prensa de papel-por parte de los sujetos con diversidad funcional, y más aún, de aquellas personas que tienen algún tipo de discapacidad intelectual o del desarrollo. En esta situación, la radio y más concretamente la radio en internet, surge como un instrumento que permite a esferas ciudadanas en riesgo de exclusión social ejercer como prosumidores (Sabés-Turmo; Parra-Valcarce, 2014) con la idea de dar visibilidad a sus pensamientos y realidades cotidianas y cambiar la imagen pública de la discapacidad.

Ante la limitada presencia de las personas con discapacidad en los medios y frente a una representación social en ocasiones desajustada, desde el asociacionismo se ha abogado por implementar medidas proactivas que pasan por apropiarse de la tecnología (Echeverría, 2008) para incrementar la aparición en los nuevos medios digitales en una nueva era, la de la sociedad de la información integradora, en la que empieza a emerger con fuerza el concepto de "infoinclusión".

El principal propósito de esta investigación es analizar una realidad reciente y creciente en el ámbito de la comunicación radiofónica y de la sociología de la discapacidad: la participación de colectivos de personas con discapacidad intelectual (en adelante, DI) o del desarrollo en espacios radiofónicos con presencia digital a través de los talleres de radio.

Para ello nos planteamos realizar una investigación en dos fases. En la primera, el principal objetivo era analizar la rela- ción entre la radio y la discapacidad para llegar a demostrar la importancia y también la necesidad de la aparición de los talleres de radio. Así, analizamos el ADN de la radio y su adaptación a las personas con discapacidad, relatamos las primeras experiencias radiofónicas relacionadas con la salud mental y abordamos la presencia de las personas con discapacidad en la radio española, focalizada en las grandes cadenas de radio, la radio municipal y la radio comunitaria o cultural.

Posteriormente, decidimos analizar la presencia de talleres de radio en nuestro país a partir del trabajo realizado por un colectivo que cuenta con una dilatada trayectoria en la gestión de este tipo de formación: la Confederación Española de Organizaciones en favor de las Personas con Discapacidad Intelectual o del Desarrollo (Feaps). Diseñamos un cuestionario para recabar información cuantitativa y cualitativa sobre la antigüedad de los centros, el tipo de difusión de sus emisores, y las tareas y roles profesionales que desempeñan las personas con DI o del desarrollo. De este modo podíamos tener una foto fija de la presencia y la organización de los talleres en toda España.

En la segunda fase de este trabajo se planteó realizar una investigación etnográfica con el objetivo de describir y examinar la participación de las personas con DI o del desarrollo inscritas en talleres de radio, así como de descubrir los significados que le dan a ese comportamiento realizado (Álvarez-Gayou-Jurgenson, 2003) ya que el principal objetivo es conocer la experiencia derivada de la intervención en espacios radiofónicos para examinar los beneficios, modo de participación y recompensas que el medio radio proporciona a este colectivo. En este sentido, partimos de la hipótesis de que la radio, como medio de información, formación y entretenimiento puede ser una excelente herramienta para satisfacer las necesidades abstractas de las personas con DI o del desarrollo que tienen que ver con el plano psicológico o afectivo (reforzar su identidad personal y sentirse bien) y con el objetivo de visibilizar sus realidades (inclusión y empoderamiento social).

La presente investigación, por tanto, se enmarca dentro del enfoque funcionalista de los "usos y gratificaciones", cuyos postulados se fundamentan en la tesis de que el público usa los medios para satisfacer sus necesidades, lo cual implica un comportamiento participativo y activo.

Así, de acuerdo con McQuail, Blumler y Brown (1972), los principales usos y gratificaciones de la radio para las personas con discapacidad intelectual o del desarrollo se basan en:

- refuerzo de la identidad personal: empoderamiento; 
- vigilancia: mantenerse informado con sentido crítico;

- relaciones sociales: inclusión e interacción;

- diversión: el ocio entendido como un fin en sí mismo.

Del mismo modo, según la clasificación propuesta por Katz, Gurevitch y Haas (1973), las necesidades que pueden ser satisfechas a través de la participación en el taller de radio serían:

- cognitivas: adquisición o refuerzo de cultura;

- estético-afectivas: los medios proporcionan una experiencia emotiva pero también estética;

- de evasión: entretienen, liberan tensiones;

- integradoras: en el plano de la personalidad permiten construir un estatus de personas informadas, y en el plano social refuerzan la interacción con personas y amigos.

La metodología utilizada es cuali-

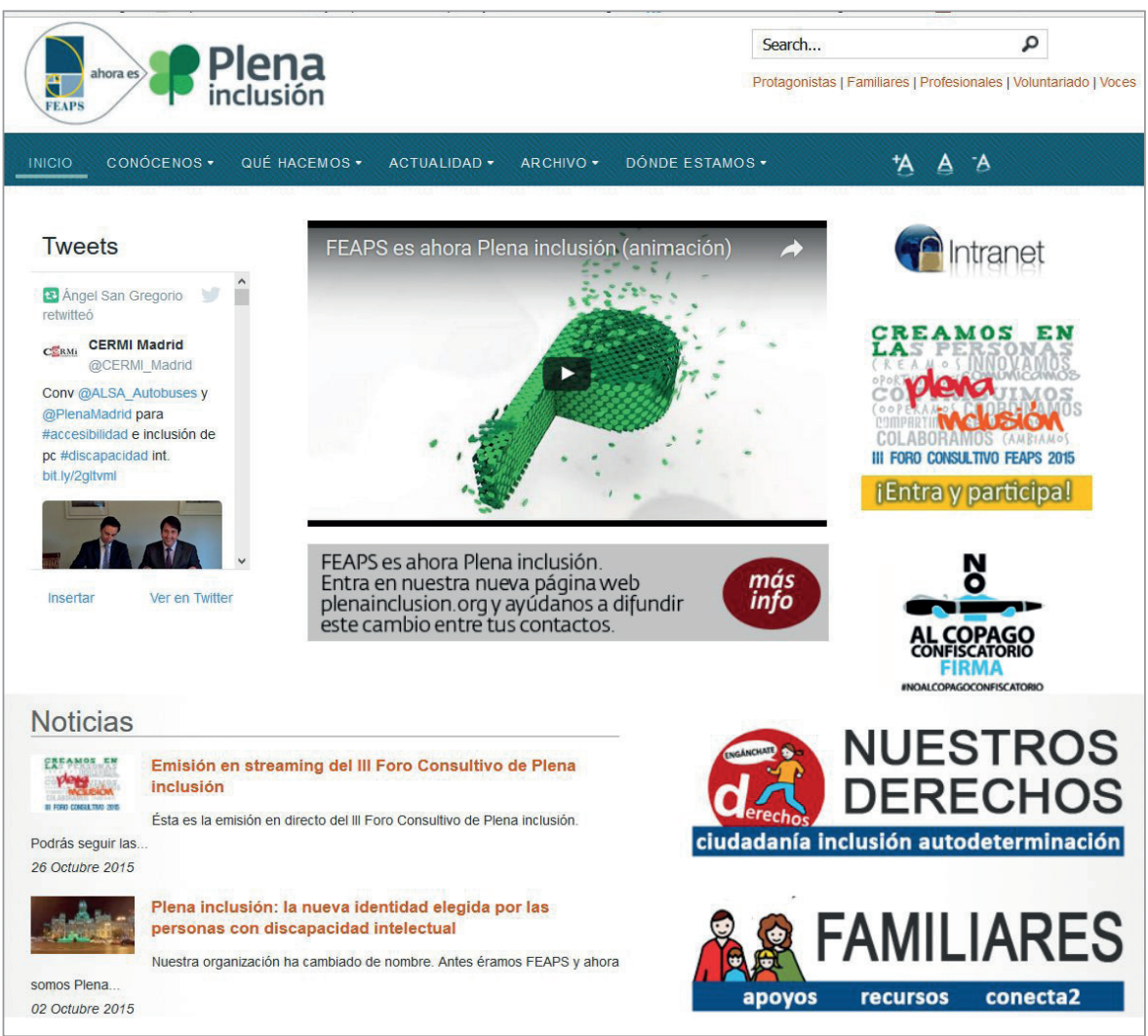

http://www.feaps.org

tativa y descriptiva, ya que lo que

se busca es captar y reconstruir significados (Ruiz-Olabuénaga, 2012) y, como afirma Báez-Pérez-de-Tudela, es la más adecuada para

"contextualizar e interpretar, en términos culturales concretos, las actitudes y motivaciones de los distintos grupos sociales" (2009, p. 38).

No en vano la investigación cualitativa parte del supuesto básico de que el mundo social está construido de significantes y símbolos, y el conocimiento es construido socialmente por las personas que participan en la investigación, de modo que la tarea fundamental del investigador es entender el mundo complejo desde el punto de vista de quienes tienen una experiencia vivencial (Mertens, 2015).

En este sentido, el diseño de la investigación cualitativa pretendía cubrir los siguientes objetivos:

- Encuadrar en el estudio los puntos de vista de los participantes (tutores y usuarios de los talleres de radio) para conocer la influencia que ejerce el medio radiofónico en este colectivo, mediante la recopilación de valoraciones y datos facilitados por los sujetos que participan de una manera habitual en talleres de radio.

- Los datos debían recolectarse en el entorno en el que las personas realizan sus actividades cotidianas. Como el objetivo era llegar al mayor número de personas en varias partes del territorio nacional, se prefirió utilizar la técnica del cuestionario distribuido desde los propios centros, en lugar de la entrevista en profundidad o el grupo de discusión. Se enviaron dos cuestionarios, uno para ser cumplimentado por los tutores, y otro para que éstos lo distribuyeran entre los usuarios de los talleres.
- Inquirir cuestiones cerradas pero también abiertas, ya que se aspira a averiguar cómo se articula la participación en los talleres y detectar qué gratificación obtienen las personas con DI o del desarrollo con su implicación. Lo que se pretende estudiar son fundamentalmente percepciones, cuya esencia no solamente se captura a través de mediciones.

La participación en talleres está motivada por la satisfacción de determinadas necesidades que tienen que ver con el plano psicológico o afectivo

Los resultados alcanzados en esta investigación permiten inferir que la presencia de espacios sobre discapacidad en la radio es aún insuficiente -salvo en la radio de proximidad-pero que se abre un amplio abanico de posibilidades para la visibilidad de las personas con DI a través de los talleres de radio ofrecidos por asociaciones como Feaps, objeto de nuestro estudio. Queda demostrado que el taller es un agente activo que, mediante la participación, promueve el empoderamiento personal y se convierte en una herramienta terapéutica para trabajar la autoestima personal del individuo con discapacidad intelectual o del desarrollo.

\section{La radio, escenario de intervención social}

La radio tiene un claro poder para visibilizar existencias ocultas. Es también una herramienta para empoderar a sus participantes y un potente agente alfabetizador mediático: educa en los medios desde una visión crítica. La radio está hecha para el servicio: informar, entretener, formar e intervenir socialmente. 


\subsection{Radio y salud mental}

Para buscar antecedentes en el campo de la intervención social a través de la radio debemos remontarnos al inicio, hace más de 20 años, del proyecto La Colifata (Argentina) -dirigido a personas con problemas de salud mental-. La finalidad de estas iniciativas es la rehabilitación personal (terapia) y la lucha social contra los prejuicios -la deconstrucción del tradicional discurso estigmatizante-. Así, las experiencias en este ámbito se han multiplicado en América Latina y en Europa.

Además de La Colifata, iniciada por el psicólogo Alfredo Olivera, se han asentado proyectos -la mayor parte de ellos integrados en la red SoVo- como:

- Radio Los inestables (Argentina)

- FM desate (Argentina)

- Radio Radiola (Argentina)

- Radio Podemos volar (Costa Rica)

- Radio Diferencia (Chile)

- Radio Vilardevoz (Uruguay)

- Radio Abierta (México)

- Radio Microsillons (Francia)

- Radio Citron (Francia)

- La terra è blu (Italia)

- Radio SoVo Polska (Polonia)

- Radio Aurora-A outra voz (Portugal)

- Radio Totalnormal (Suecia)

A partir del movimiento pionero creado por la asociación cultural Radio Nikosia, en España surgieron:

- Onda cerebral (Hospital Psiquiátrico Penitenciario de Sevilla)

- Radio travesías (Marbella, Málaga)

- Remolinos de quijotes (El Ejido, Almería)

- Romper barreras (Málaga)

- Radio naranja (Mallorca)

- Radio divergente (Salamanca)

- Radio Morea (Orense)

- Onda positiva (Getafe, Madrid)

- Radio tarumba (Madrid)

- Mejor imposible (Madrid)

- Ábrete camino (Madrid)

- Frecuencia favorable (Madrid)

Sólo en la comunidad de Madrid, como señalan Fernández-Sande y Leal-Leal (2015), se han contabilizado en los últimos años hasta 20 proyectos radiofónicos.

Estas iniciativas son experiencias de intervención en la comunidad que buscan cambiar la mentalidad social acerca de los enfermos de salud mental y propiciar una terapia rehabilitadora que mejore la calidad de vida de las personas participantes.

http://www.vivalacolifata.org

\subsection{Discapacidad y radio social}

Las grandes cadenas de radio apenas se ocupan de la temática de la discapacidad. Lejos quedan en el tiempo programas como

- Un mundo sin barreras, de la ONCE (Onda Cero), con casi tres lustros de existencia;

- El club de la vida (RNE), que estuvo en antena casi 20 años.

Más recientes son espacios enmarcados en grandes magacines radiofónicos como

- Ser capaces (Hoy por hoy, Cadena Ser);

- Mundo social (Herrera en la Onda, Onda Cero).

En los últimos años, la tendencia dominante ha sido la desaparición de programas y espacios de las parrillas de programación. Así, y según la consulta realizada a través de las webs de las principales empresas radiofónicas españolas y de la realización de entrevistas a los directores de comunicación de las cadenas privadas Cope, Ser y Onda cero (realizada por los autores en enero de 2016), se pone de manifiesto que estas emisoras carecían de programas dedicados a la discapacidad en la temporada 2015-2016.

En Onda cero, el tratamiento informativo en programas se reducía a informaciones en secciones sobre salud (En buenas manos) o sobre historias de superación y enfermedades raras (Te doy mi palabra, Más de uno).

En la Cadena Ser, los temas sociales se canalizaron a través de las plataformas programáticas de mañana, tarde y madrugada (Hoy por hoy, La ventana, Hablar por hablar).

La Cadena Cope era la única cadena de radio privada nacional con secciones específicas sobre discapacidad. El partido de las 12, programa deportivo nocturno, emitía los lunes el espacio Cómo lo ves, protagonizado por tres deportistas ciegos (Antonio, Vicente y José) que hablaban de las noticias deportivas desde su perspectiva personal.

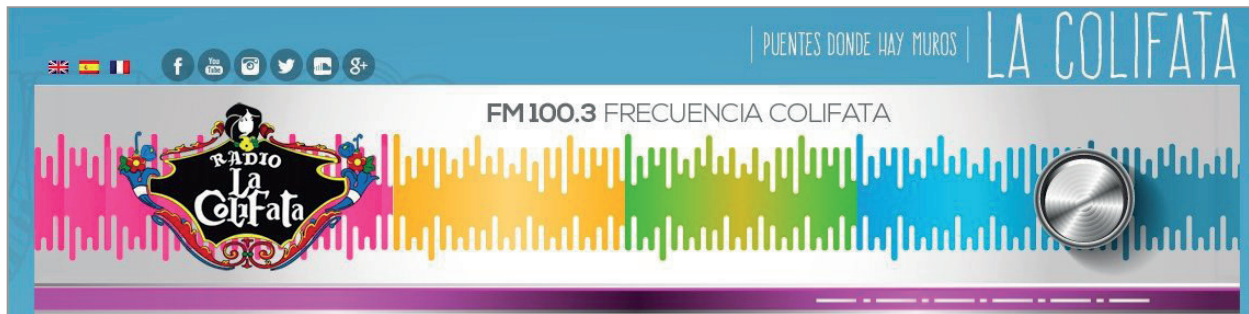

INICIO INSTITUCIONAL COLFATARADIO NOTICIAS COLFATAS MULTIMEDIO COLFATO EVENTOS OTRAS COLIFATAS COLIFATA FRANCIA CONTACTO

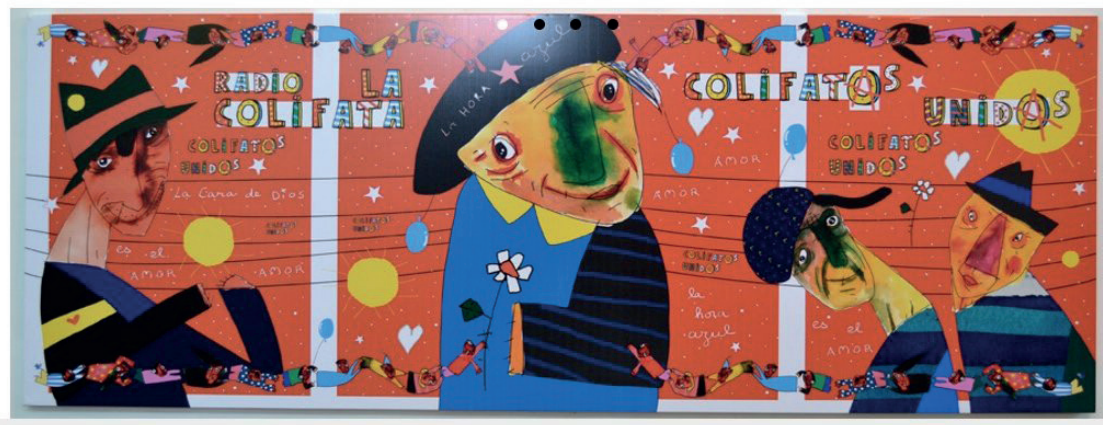


En otros programas como La tarde, Herrera en Cope o Fin de semana la discapacidad era abordada como asunto de actualidad esporádicamente. Un caso excepcional es el programa La noche, que emitía relatos de personas con enfermedades raras y discapacidades con una periodicidad semanal.

Si nos acercamos a la radio pública, comprobamos la existencia de programas como:

- Juntos paso a paso (RNE, sábados de 07:00 a 08:00);

- el micro-espacio Descubriendo capacidades (Radio 5, miércoles a las 18:47);

- Si se quiere, se puede (Radio 5, jueves de 00:35 a 01:00);

- Para que veas (Radio 5, de lunes a viernes a las 11:47);

- Sin fronteras (domingos, de 13:40 a 14:00 en RNE-Comunidad Valenciana).

Se observa que la presencia de programas o secciones de programas en las grandes cadenas de radio es aún marginal. Tan sólo la radio pública dispone de espacios específicos. Estos datos nos llevan a reflexionar acerca de la necesidad de que las personas con diversidad funcional tengan una mayor aparición en las parrillas de la radio. Por su peso poblacional y por constituir un heterogéneo grupo humano en riesgo de exclusión social, el colectivo de la discapacidad merece una mayor exposición pública.

La radio es un medio muy útil para construir una sociedad de la información integradora e infoinclusiva

\subsection{Espacios extramuros: la discapacidad en la radio local}

La radio local (tanto la comunitaria-cultural como la autonómica y municipal) ha mostrado una mayor sensibilidad ante la discapacidad. Estamos ante un concepto de radio social (Contreras-Pulido; González-Mairena; Aguaded-Gómez, 2014) al servicio de la ciudadanía que defiende el criterio de la diversidad en la programación. Dentro de esta definición, y tal y como señalan investigadores como Mateo-Mateo (2010) o Díaz-Aledo (2007), en los últimos años se han emitido programas en el ámbito regional como

\footnotetext{
- Andalucía sin barreras (RTVA, Canal Sur Radio)

- Discapacidad sin distancias (Onda Vasca)

- Convivir en igualdade (Radio Galega)

- Descubriendo capacidades (RNE Cantabria)

- Catalunya sense barreres (Ràdio Estel).
}

Entre los espacios de alcance provincial encontramos ejemplos como:

- La discapacidad en el siglo XXI, dirigido por el periodista con discapacidad visual Pedro García-Recover en Punto Radio Málaga;

- Nosotros también (La Telaraña Radio);

- Torrelavega solidaria (Onda Cero Torrelavega).

En emisoras comunitarias son reseñables las aportaciones de Radio Vallekas.

Si acercamos la lupa a propuestas singulares, en 2011 comenzó a funcionar en Toledo (Hospital Nacional de Para- pléjicos) la emisora Onda Discapacidad (emisión en internet), que cuenta con la colaboración de investigadores del centro, mientras que en 2013 se iniciaron las emisiones de Radio Disc (Huelva), una radio hecha por personas con discapacidad que emite en FM y en la Web. http://www.radiodisc.com

Mención especial merecen dos cadenas de radio:

Libertad FM (Comunidad de Madrid), en la que se han emitido programas como:

- Bienvenidos a la radio

- Escaleras de la dependencia

- La barandilla deportiva

- Mentes peligrosas (presentado por el humorista Mariano Mariano).

Gestiona radio, en la que se ha emitido en los últimos tiempos Gestiona social (agencia Servimedia), presentado por Arturo San Román.

En general, el hueco que dejan las radios de corte generalista es ocupado -aunque su incidencia en número es aún limitada-, por las radios autonómicas, emisoras de provincias de las cadenas generalistas, emisoras locales públicas, emisoras universitarias y emisoras comunitarias.

\section{El binomio radio/discapacidad}

La radio tradicional alberga rasgos de identidad que pueden contribuir a mejorar los procesos de comunicación de las personas con DI o del desarrollo. Es un medio fugaz y caliente (Ortiz; Volpini, 1995), rápido y económico (MuñozGonzález; Gil, 1994), caracterizado por la temporalidad (sonido unido al tiempo), la linealidad (visión secuencial) y la irreversibilidad del sonido (sonido siempre efímero) (Merayo-Pérez, 2000), por su alta presencia en la sociedad y sus sencillas características técnicas (Herrera-Damas, 2003).

Aunque estos rasgos forman parte del ADN del medio radio, el núcleo de su dimensión comunicativa y expresiva es la preeminencia de la palabra, procedente del hecho cultural de la oralidad (Cebrián-Herreros, 1994).

En una sociedad en la que prima la imagen, la radio nos propone como referente el valor del sonido. Está hecha para el oído, dice Rodero (2008, p. 106):

"el canal que se sustenta por completo en el lenguaje oral despojado de cualquier recurso icónico".

Es un medio ciego en el que el individuo con DI o del desarrollo se siente acogido y, hasta cierto punto, protegido, aislado de la gran aldea de lo visual en la que los sujetos son escrutados y juzgados por su apariencia en base a unos clichés instalados en la comunidad.

Aunque la 'ceguera' de la radio es una presunta discapacidad frente a otros medios de comunicación, la radio ha sabido acoger la magia del sentido auditivo para elevar la palabra hablada a su expresión más genuina. Así, el escuchante asimila un concepto de comunicación unisensorial en el que es posible la seducción o, por el contrario, la distancia a través de la semiótica de la palabra, de la música, de los efectos y del silencio. La radio busca contener una emoción estética, pretende emocionar (Balsebre, 1994). En cierto modo, esa 
comunicación afectiva, ese sonido erigido en emoción (Kaplún, 1998) provoca una ruptura con el hermetismo derivado de la timidez y la falta de confianza personal, actitudes frecuentes en las personas con DI o del desarrollo.

Por otra parte, y desde una óptica económica, la radio es un medio viable. En la era digital, los servicios de alojamiento de podcasts ofrecen un yacimiento de oportunidades para abrirse paso en el fenómeno de la comunicación radiofónica. Además, su sencillez técnica contribuye a que los colectivos con mayores dificultades intelectuales tengan una diligente adaptación al medio. En síntesis, internet ha democratizado el acceso a la creación de servicios y productos en la radio.

\subsection{Radio en la Red y empoderamiento ciudadano}

En una sociedad claramente marcada por la transversalidad de la tecnología como elemento diferenciador de nuestro tiempo y caracterizada por la desigualdad en el acceso a las TIC -brecha digital- y en el aprovechamiento de sus recursos, resulta urgente la alfabetización digital. Sólo así podremos minimizar el hecho real de la exclusión social -ahora tecnológica-.

En este contexto, en el que cualquier contenido en internet tiene una potencialidad universal o global y, al mismo tiempo, penetra en lo local, es preciso dar voz a las personas más vulnerables, facilitar la accesibilidad a los medios.

En el entorno de la alfabetización mediática, las TIC son un motor de impulso que contribuyen a visibilizar la realidad de los colectivos en riesgo de exclusión social, propiciar nuevas interconexiones sociales y potenciar a los individuos a través de la educomunicación pues, como dice Castells (1995), vivimos en una mediocracia en la que el impacto de los medios de comunicación alcanza a todos los niveles.

Esto será posible comprendiendo los conceptos de participación y empoderamiento. En el entorno de la discapacidad, la idea de participación fue impulsada en la Clasificación internacional del funcionamiento, de la discapacidad y de la salud (OMS, 2001) y en el 11․ Manual de la American Association of Intellectual Disability and Developmental Disabilities (Shalock et al., 2011). Según este manual, la participación está ligada al funcionamiento de la persona, a los modos en que interacciona mediante el desarrollo de actividades en el corpus social: educación, ocio, cultura, vida espiritual o trabajo.

La participación en un espacio radiofónico puede ayudar a la persona con DI o del desarrollo a darse cuenta de que, pese a las diferencias en la realización de algunas habilidades con otros colectivos, y, quizá, la carencia de oportunidades en el plano social, existen cauces para tomar conciencia de sus capacidades y desplegar ciertos talentos que pueden pasar desapercibidos.

Sin duda, la participación en la sociedad -vía medios de comunicación- es un buen aliado para el empoderamiento de las personas con discapacidad, pues refuerza las capacidades del individuo, mejora el control de su vida y posibilita una mayor influencia social (Rappaport, 1987).

La radio en la Red, en cuanto TIC accesible, es una plataforma idónea para encajar el concepto de participación y empoderar a las personas con discapacidad. Permite la incubación de redes, el intercambio de experiencias y de información y la creación de lugares de encuentro (Jiménez-Lara, 2009).

En cierto modo, asumida la realidad de que la atención a la discapacidad desde las grandes plataformas mediáticas es muy escasa, los colectivos de personas con diversidad funcional han comenzado a adoptar un papel proactivo y diseñar proyectos propios de comunicación (Sánchez-de-Amo, 2003) que se convierten en altavoces de sus intereses. En esta línea, encontramos la revista especializada, el taller de televisión o vídeo, o el taller de radio. El uso de las plataformas de alojamiento de radios online y servicios de podcasting es un mar de posibilidades que garantiza la accesibilidad radiofónica. En esta esfera, la convergencia con internet ha suscitado la aparición de la webradio o ciberradio, que se presenta como una oportunidad de acceso a los medios audiovisuales y como una gran plataforma para alfabetizar mediáticamente. De hecho, ha incrementado las posibilidades de creación en el universo radiofónico dando lugar a un mayor número de canales con la aspiración de consolidar audiencias heterogéneas. En consecuencia, permite erigir un medio de comunicación propio para un público interesado en contenidos concretos (Cebrián-Herreros, 2009).

La participación en un taller de radio puede ayudar a la persona con discapacidad intelectual o del desarrollo a tomar conciencia de sus capacidades y desplegar talentos desapercibidos

Estas nuevas herramientas o "nanomedios" (Escandell-Montiel, 2015) son útiles porque pueden ayudar al ciudadano a escapar del control de la información que ejercen los grandes grupos dedicados al negocio del periodismo y el entretenimiento, a construir y difundir sus mensajes en libertad. La revolución tecnológica obliga, dicen Contreras-Pulido y Aguaded (2012), a repensar la comunicación dentro de un nuevo paradigma. Una comunicación que ofrece a las personas con DI o del desarrollo una multiplicidad de afluentes informativos y culturales, redes para el aprendizaje, el diálogo y la participación, y que dispensa la potencialidad de crear micromedios para empoderarse mediáticamente.

En suma, la radio en internet puede ser una palanca de intervención social capaz de conquistar espacios de expresión, visibilidad social y comprensión crítica de la comunicación para reforzar la libertad de expresión y el derecho a la información, además de robustecer la ética de las sociedades.

\section{Metodología}

Como ya hemos explicado anteriormente, en esta investigación se han triangulado técnicas cuantitativas y cualitativas de recogida de datos dentro de una metodología descriptiva.

Tras un proceso de recopilación de información mediante cuestionarios y entrevistas telefónicas con los directores de comunicación de las federaciones regionales y en las delegaciones provinciales de Feaps, se recabaron datos de 38 talleres radiofónicos en los que participan personas con DI o del desarrollo, lo que constituye el núcleo de una primera 
base de datos de este tipo. La media de participación en dichos talleres se acercaba a los 600 usuarios.

El período de recogida de datos fue en dos momentos: entre enero y julio de 2014 y entre octubre de 2014 y enero de 2015. Tras un primer contacto telefónico, se elaboró y envió un cuestionario -vía correo electrónico- que debía ser contestado por los tutores de los proyectos de radio con preguntas cerradas y abiertas. Ese cuestionario pretendía trazar un perfil sobre el funcionamiento de los talleres:

- situación geográfica;

- media de participantes;

- tipo de emisión: directo/grabación, FM/internet/interna;

- tipo de emisoras: privadas, públicas, comunitarias;

- tareas realizadas en los talleres por los usuarios, tutores y profesionales de las radios colaboradoras y competencias; - objetivos de los talleres radiofónicos.

En una segunda fase se remitió un nuevo cuestionario validado por un grupo de ocho expertos- a los usuarios de los talleres - personas con DI o del desarrollo- para obtener información acerca de los usos que hacen de la radio y las gratificaciones que obtienen a cambio. Como el objetivo era llegar al mayor número de participantes que estaban dispersos por toda la geografía española, se utilizó la técnica del cuestionario distribuido desde los propios centros, en lugar de la entrevista en profundidad o del grupo de discusión.

Se envió la encuesta a los tutores para que éstos la distribuyeran entre los usuarios de los talleres. Constaba de tres partes:

- rasgos sociodemográficos de los participantes en los talleres;

- papel y características de los tutores;

- análisis de la influencia del taller en las personas con DI o del desarrollo.

Para realizar la muestra de este segundo cuestionario se consideró un error muestral igual a +/-5\% para 238 entrevistas. El nivel de confianza (K) era del 95,5\%. Finalmente, y tras enviar la encuesta a los 38 talleres de la base de datos, contestaron 175 de los casi 600 usuarios participantes, en torno a un $30 \%$ del total de alumnos. La edad media de quienes participaron en la segunda encuesta es de 40,2 años.

\section{Análisis e interpretación de resultados}

\subsection{Antigüedad de los talleres: la influencia de internet}

De los 34 talleres que se pueden encontrar en internet, 29 disponen de servicio de podcast y 26 se pueden escuchar online (a través de streaming o en web). Más de la mitad de estos talleres (22) disponen simultáneamente de emisión online y servicio de alojamiento de podcasts.

Uno de los datos más relevantes del estudio es que la mayoría de los proyectos de participación en la radio son recientes, casi las tres cuartas partes de los talleres (26 de 38) tienen una antigüedad inferior a los cinco años.

El $26 \%$ del total de proyectos con menos de cinco años se produce en Madrid (10). En Andalucía, que es la segunda comunidad, hay 4 talleres (10\%), en Castilla y León se realizan 3 y en Castilla La Mancha, 2. En el resto de las regiones se ha contabilizado un taller en cada una.
En la tabla 1 estratificamos los datos concretos de este tipo de talleres de nueva creación por comunidades autónomas.

Buena parte de estos talleres con menos de cinco años de antigüedad, en torno a un 90\% (22 de 26), están presentes en internet: Haciendo amigos, Prode de cerca, Te lo contamos, Ruedas y Piernas, Radio Teatro de Feaps, Mi voz importa, Con buena onda, Capaces en la Onda, El cimbalillo de la Fundación, Voces que cuentan, La tertulia de Pláceat, La radio de los gatos, Adisleando, Radio terrícola, Yo como tú, Voces para el corazón, Radio Alegría -edición Pozuelo, Radio Alegría -edición La Granja, Radio o lo que sea, Gente extraordinaria, El trébol de 4 hojas y muchas más, y El oído del saltamontes.

\subsection{La supremacía de internet}

De los 38 talleres de la base de datos creada dentro de $\mathrm{Fe}$ $a p s$, los proyectos de radio que emitían en emisoras convencionales de frecuencia modulada (FM) eran 27. Sin embargo, y aunque una mayoría de colectivos de personas con DI

Tabla 1. Talleres con una antigüedad inferior o igual a 5 años

\begin{tabular}{|c|c|}
\hline CCAA & Nombre del taller \\
\hline \multirow{4}{*}{ Andalucía } & Haciendo amigos (Asociación Disgenil, Córdoba) \\
\hline & Prode de cerca (Asociación Prode, Córdoba) \\
\hline & Te lo contamos (Asociación Adefisal, Sevilla) \\
\hline & Ruedas y piernas (Asociaciones ASAS y Aspace, Sevilla \\
\hline Aragón & Radio teatro de Feaps (Feaps Aragón, Zaragoza) \\
\hline Baleares & Mi voz importa (Feaps Baleares, Palma de Mallorca) \\
\hline Cantabria & Radio alternativa (Asociación Amprós, Santander) \\
\hline \multirow{2}{*}{$\begin{array}{l}\text { Castilla La } \\
\text { Mancha }\end{array}$} & Con buena onda (Asociación Asprona, Albacete) \\
\hline & Capaces en la onda (Asociación Down Toledo, Toledo) \\
\hline \multirow{3}{*}{ Castilla León } & $\begin{array}{l}\text { El cimbalillo de la Fundación (Fundación San Cebrián, } \\
\text { Palencia) }\end{array}$ \\
\hline & Voces que cuentan (Fundación San Cebrián, Palencia) \\
\hline & Espacio Asprodes (Asociación Asprodes, Salamanca) \\
\hline $\begin{array}{l}\text { Comunidad } \\
\text { Valenciana }\end{array}$ & Somos uno más (Asociación Adisto, Valencia) \\
\hline Extremadura & La tertulia de Pláceat (Asociación Pláceat, Cáceres) \\
\hline Galicia & La radio de los gatos (Asociación Aspace, A Coruña) \\
\hline La Rioja & Nuestra voz (Asociación ARPS, La Rioja) \\
\hline \multirow{10}{*}{ Madrid } & Adisleando (Asociación Adisli, Madrid) \\
\hline & Radio terrícola (Asociación APMIB-Envera, Madrid) \\
\hline & Yo como tú (Feaps Madrid, Madrid) \\
\hline & $\begin{array}{l}\text { Voces para el corazón (Fundación Instituto San José, } \\
\text { Madrid) }\end{array}$ \\
\hline & $\begin{array}{l}\text { Radio Alegría, edición Pozuelo (Fundación Gil Gayarre, } \\
\text { Madrid) }\end{array}$ \\
\hline & $\begin{array}{l}\text { Radio Alegría, edición La Granja (Fundación Gil Gaya- } \\
\text { rre, Madrid) }\end{array}$ \\
\hline & Radio o lo que sea (Fundación Juan XXIII, Madrid) \\
\hline & $\begin{array}{l}\text { Gente extraordinaria (Fundación Síndrome de Down, } \\
\text { Madrid) }\end{array}$ \\
\hline & $\begin{array}{l}\text { El trébol de } 4 \text { hojas y muchas más (Fundación Trébol, } \\
\text { Madrid) }\end{array}$ \\
\hline & El oído del saltamontes (Grupo AMÁS, Madrid) \\
\hline
\end{tabular}


o del desarrollo han alcanzado acuerdos con emisoras de FM para realizar programas o secciones de programas, es notoria la influencia de internet, bien como alternativa única de comunicación radiofónica o como vía complementaria a las emisiones en FM.

En la encuesta se detectó igualmente que una cuarta parte de los talleres de radio se realizan en formato de radio interna. Es una metodología fundamentada en emisiones por megafonía o en la realización de espacios-piloto o ensayos radiofónicos. Sólo en ocasiones estos espacios grabados se reproducen con una cierta regularidad en emisoras convencionales o en internet. Es evidente que, en una época en la que dominan las TIC, es más que recomendable que los talleres de radio interna se incorporen al universo de internet con la idea de visibilizar sus realidades.

De los 38 talleres de radio, 16 se emiten en directo, otros 16 son grabados y 6 se emitían tanto grabados como en directo. De las emisiones en directo, 12 se emiten en FM, 10 tienen servicio de podcast, 12 son online y 2 son por megafonía interna.

Si nos aproximamos a las grabaciones, 9 lo hacen en emisoras de FM, 15 tienen alojamiento para podcasts, $10 \mathrm{se}$ emiten online y 2 son proyectos de radio interna. Una mayoría de las grabaciones son usadas como piezas de audio en formato podcast.

\subsection{Tareas de los talleres}

Según la consulta a los tutores, existen experiencias radiofónicas en las que cada usuario interviene asumiendo un rol determinado dentro de un proceso holístico, mientras que hay otros talleres, sobre todo aquellos en los que el nivel de participación es menor, en los que la persona con DI o del desarrollo realiza varias funciones a la vez. En general, la mayor parte de los participantes -salvo quienes no saben leer o vocalizan con excesivas dificultades- suelen trabajar ante el micrófono regularmente. En nuestra definición de tareas de los usuarios hemos registrado las siguientes figuras:

- presentación del programa o del espacio;

- elaboración del guion y la escaleta;

- colaboración en tertulias y secciones de opinión;

- realización de entrevistas;

- control de sonido;

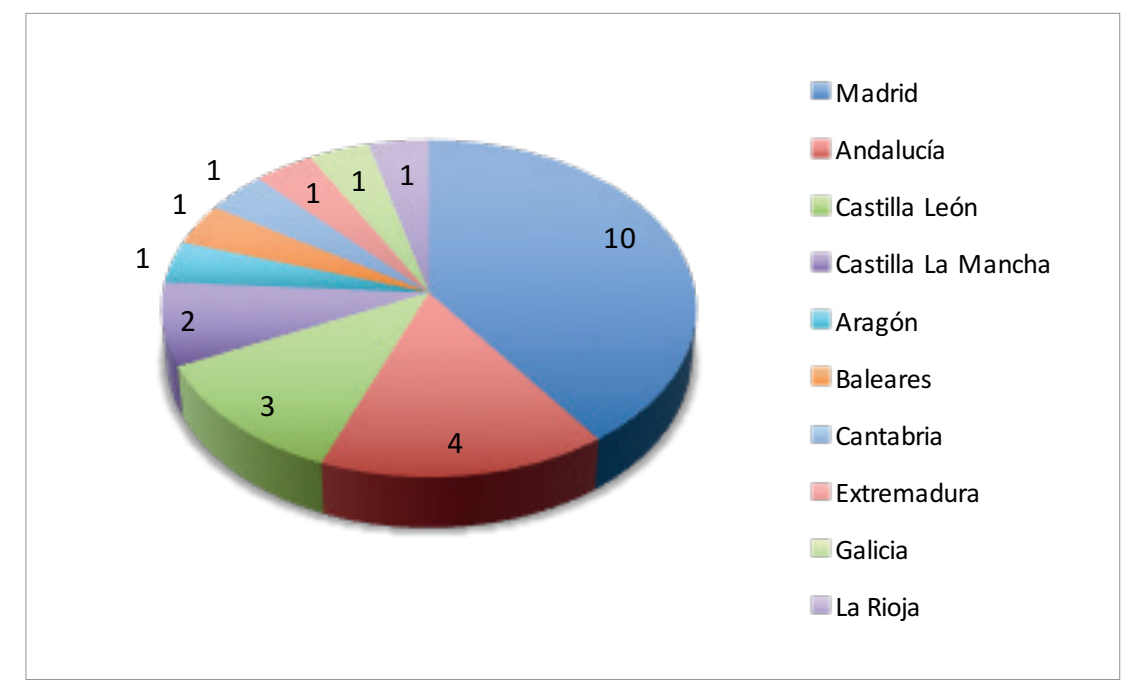

Gráfico 1. Talleres con una antigüedad inferior o igual a 5 años

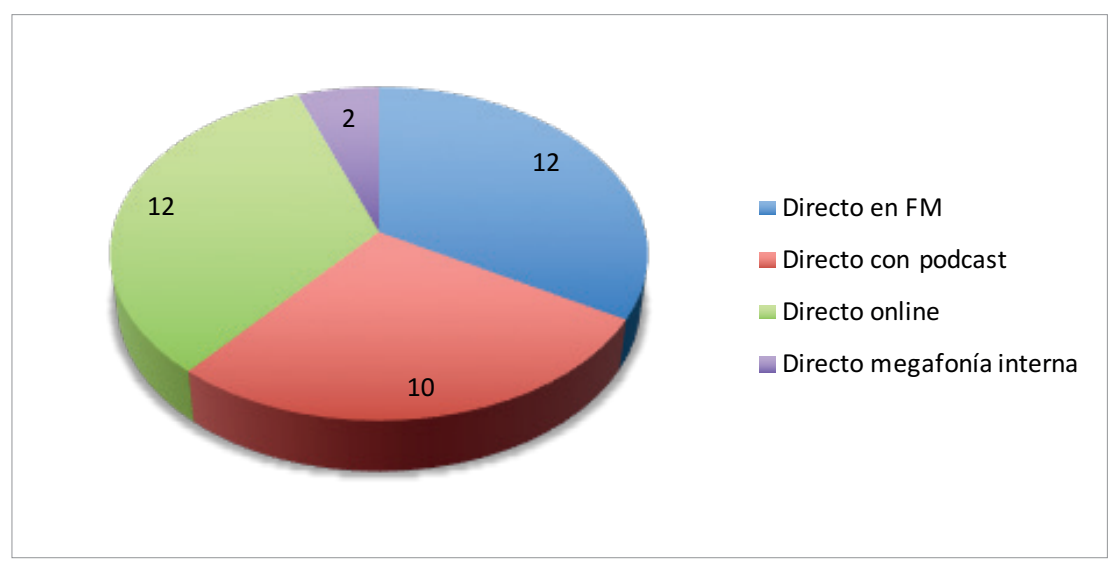

Gráfico 2. Emisiones en directo en FM, podcast, online y radio interna

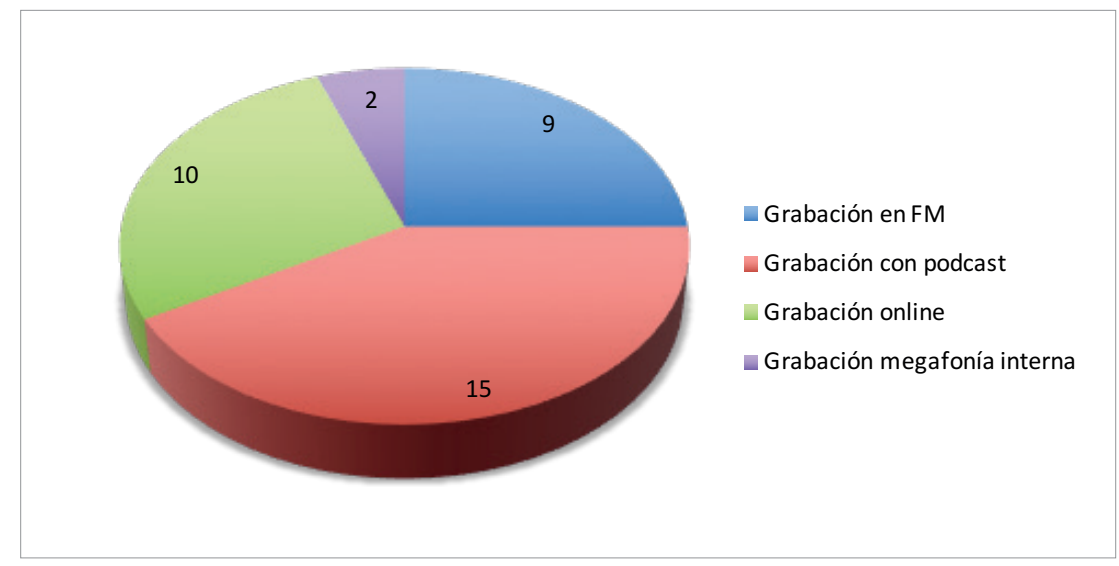

Gráfico 3. Emisiones con grabaciones en FM, podcast, online y radio interna
- redacción de noticias, reportajes y crónicas;

- locución;

- gestión web;

- producción.

El cuestionario respondido por los participantes en los talleres revelaba que la labor más realizada es la locución $(98,2 \%)$, seguida de la producción $(54,2 \%)$, redacción $(45 \%)$, presentación del espacio $(41,8 \%)$, gestión web $(13,7 \%)$ y control técnico $(8,9 \%)$. 
El tutor, que es personal de las entidades, colabora como presentador de programas o secciones, supervisor del guion, organizador de espacios de debate, colaborador en la realización de entrevistas, gestor web y coordinador de producción. El $80 \%$ de los tutores se dedica a coordinar el grupo, mientras que el $20 \%$ restante interviene en funciones de apoyo.

El personal externo a las entidades está formado por los presentadores y locutores de las emisoras en las que se emiten los espacios realizados en los talleres. Ejemplos de programas o secciones presentados por profesionales son:

- Radio alegría Pozuelo (Radio enlace);

- Radio alegría La Granja (Radio enlace);

- Capaces (Onda cero);

- Capaces en la onda (Onda cero).

En estos casos, el papel es el de meros intermediarios que intervienen esporádicamente para conducir el espacio, aunque se mantienen en un plano discreto. La coordinación técnica en los días de ensayos y grabaciones y, en menor medida, la gestión web son otras tareas realizadas por los profesionales radiofónicos.

El taller de radio promueve el empoderamiento personal y se convierte en una herramienta terapéutica para trabajar la autoestima personal del individuo con discapacidad intelectual

\subsection{Motivos de participación de los usuarios y obje- tivos de los talleres}

El principal motivo de participación, en opinión de los usuarios, es la experiencia de felicidad que proporciona la participación. Utilizando una escala tipo Likert del 1 (totalmente de desacuerdo) al 10 (totalmente de acuerdo) ésta fue la respuesta más elevada con un 8,62 de puntuación media. La segunda razón esgrimida es el disfrute de un tiempo de ocio y entretenimiento $(8,36)$. El tercer motivo elegido está relacionado con la necesidad de relacionarse más y mejor $(8,15)$. A los usuarios les interesa también (cuarto motivo en puntuación) que el entorno les conozca mejor $(8,12)$. Los ítems "Participo para hablar mejor" y "Participo para mejorar la escritura" fueron los menos apreciados (obteniendo un 7,4 y 5,3 , respectivamente).

Desde el punto de vista de los tutores el principal motivo para crear estos talleres es visibilizar la discapacidad y trasladar una imagen de normalización a la sociedad (95\% de las respuestas recibidas), lo cual entronca con el principal objetivo de las asociaciones de colectivos desfavorecidos: buscar una representación en la sociedad para erradicar estigmas y mostrar una imagen de inclusión.

El segundo objetivo que cumple el taller para los tutores es "Empoderar a las personas con discapacidad a través del trabajo de la autoestima, el desarrollo de habilidades comunicativas y sociales" (92\%) y le siguen "Analizar la realidad con espíritu crítico (educomunicación)" (87\%) y el "Fomento del ocio y tiempo libre" (85\%).
Como puede apreciarse, hay bastante convergencia entre los objetivos expuestos por la organización y los motivos de la participación de los usuarios, aunque el punto de vista sobre el orden de valoración es diferente para los dos colectivos (tutores y usuarios).

La presencia de espacios sobre discapacidad en la radio es aún insuficiente, salvo en la radio de proximidad

\subsection{Grado de satisfacción con la actividad}

Tras haber conocido las motivaciones para participar en los talleres de radio, la pregunta acerca del grado de satisfacción con la actividad, realizada a modo de escala del 1 al 10 , nos muestra que el valor máximo 'Muy contento', fue elegido por un $67,3 \%$ de los encuestados. La suma de los valores 8,9 y 10 es del $90,5 \%$ y sólo un $1,2 \%$ de los usuarios ha escogido valores por debajo del 5 . Con estos datos se comprueba que el grado de satisfacción es muy alto ya que la media alcanzada es de 9,24 sobre 10 .

En cuanto a la pregunta sobre cómo de importante consideran la presencia del taller en sus vidas en una escala del 1 al 5 (de 'Muy importante' a 'Nada importante') el 56,7\% de los usuarios asegura que es 'Muy importante'. Además, si sumamos las tres respuestas de índole positiva que se ofrecían en el cuestionario ('Muy importante', 'Importante' y 'Bastante importante') el porcentaje alcanza el 97,6\% lo que demuestra que, de acuerdo con la principal aspiración de los usuarios de participar para sentirse más felices, el taller de radio es una experiencia vivencial personal influyente.

Para concluir la exposición de resultados podemos decir que la radio en general y, más concretamente, la radio en internet, es un medio muy útil para construir una sociedad de la información integradora e infoinclusiva y que, además, debe garantizar, como apunta la ONU (2008) en la Convención sobre los derechos de las personas con discapacidad (art. 9), la accesibilidad a la información y las comunicaciones, y el derecho a la libertad de expresión y opinión. Por otra parte, la participación, el desempeño de la persona en actividades reales en ámbitos de la vida social que se relaciona con su funcionamiento en la sociedad (asunción de roles e interacciones en diferentes contextos), se reafirma como un pilar fundamental del modelo multidimensional del funcionamiento humano (Shalock et al., 2011).

\section{Conclusiones}

Tras analizar los resultados obtenidos en la presente investigación se comprueba que la presencia de programas o secciones dedicadas al mundo de la discapacidad en los medios audiovisuales es aún marginal. Según el análisis realizado sobre las programaciones de las principales cadenas generalistas españolas en la temporada 2015/2016 sólo el grupo RTVE contaba con algunos espacios específicos. En el resto de cadenas el tratamiento de la discapacidad es algo ocasional. Sin embargo, la radio de proximidad, emisoras autonómicas públicas y privadas provinciales o regionales y 
municipales han incrementado el número de espacios dedicados a la discapacidad.

Respecto al análisis y la recopilación de datos sobre los talleres de radio que se imparten en la Confederación Española de Organizaciones en favor de las Personas con Discapacidad Intelectual o del Desarrollo (Feaps), el estudio revela la existencia de 38 proyectos distribuidos geográficamente en 20 provincias de 13 regiones españolas con una media de participación cercana a los 600 usuarios. De ellos, 34 talleres tienen presencia en internet a través de servicios de podcasting y/o emisión online, frente a las tres cuartas partes ( 27 talleres) que emiten en FM. El $43 \%$ de estos talleres tienen una antigüedad de 2-3 años mientras que sólo el 20\% lleva una trayectoria mayor de 6 años, por lo que se puede deducir que las TIC han contribuido a generar nuevas experiencias radiofónicas así como el interés de los usuarios por formar parte de estos proyectos.

La radio tiene un claro poder para visibilizar existencias ocultas y educa en los medios desde una visión crítica

El estudio cualitativo, cuya pretensión era conocer las percepciones que tienen las personas con DI o del desarrollo sobre su participación en los talleres siguiendo el modelo de la teoría de usos y gratificaciones, corrobora que las principales motivaciones que justifican la participación de las personas con DI en estos proyectos radiofónicos es la satisfacción de determinadas necesidades que presentan puntuaciones muy similares (en torno a 8 sobre 10). Estas motivaciones son, por este orden:

- la experiencia emocional ("Participo para sentirme más feliz");

- la diversión ("Participo porque es un tiempo de ocio");

- las relaciones sociales ("Participo para relacionarme más y mejor");

- el refuerzo de la identidad personal ("Participo para que se me conozca mejor").

En cambio, los usuarios apenas puntuaron el desarrollo de habilidades comunicativas (hablar mejor o mejorar la escritura). Esto es así a pesar de que la principal tarea que desempeñan los usuarios es la locución (ejercida por el $98 \%$ de los participantes) y de que la tarea de hablar sea la más atractiva para ellos, ya que es la preferida frente a otras como la producción o la redacción (45\%).

Las motivaciones expuestas por los usuarios entroncan con las necesidades expuestas por Katz, Gurevitch y Haas (1973):

- de evasión-diversión, de información y comprensión de la realidad:

- de desarrollo afectivo-emocional, de mejora de las relaciones sociales y refuerzo de la identidad.

También se corresponden, aunque en orden diferente, con los objetivos que buscan los tutores con la puesta en marcha de estos talleres:

- visibilizar la discapacidad mostrando una imagen de inclusión a la sociedad;
- empoderar a las personas con discapacidad a través del refuerzo de la identidad personal con el trabajo de la autoestima y el desarrollo de habilidades sociales y comunicativas.

Se pone así de manifiesto, como señala la teoría de usos y gratificaciones, que la mera exposición a los medios de comunicación (el continente) puede resultar tan gratificante, como pueden serlo los contenidos.

El taller de radio se presenta como una experiencia comunicativa que puede ser terapéutica para trabajar la autoestima personal del individuo con discapacidad intelectual o del desarrollo. Puesto que la apropiación de la tecnología radiofónica se produce en un escenario de pertenencia grupal, se genera un encuentro con la sociedad, y estos factores benefician la inclusión y normalización. Además, el taller se concibe como una experiencia de ocio en sí misma, al favorecer la expresión artística, la creatividad y el aprovechamiento del tiempo libre.

\section{Bibliografía}

Álvarez-Gayou-Jurgenson, Juan-Luis (2003). Cómo hacer investigación cualitativa: fundamentos y metodología. México: Paidós. ISBN: 9688535168

Báez-Pérez-de-Tudela, Juan (2009). Investigación cualitativa. Madrid: ESIC. ISBN: 9788473565998

Balsebre, Armand (1994). El lenguaje radiofónico. Madrid: Cátedra. ISBN: 9788437621777

Casado, Demetrio (2001). Comunicación social en discapacidad. Cuestiones éticas y de estilo. Madrid: Ministerio de Trabajo y Asuntos Sociales; Real Patronato sobre Discapacidad. https://goo.gl/Iqm5mJ

Castells, Manuel (1995). "La mediocracia”. El país, 24 enero. https://goo.gl/D31HOZ

Cebrián-Herreros, Mariano (1994). Información radiofónica: Mediación técnica, tratamiento y programación. Madrid: Síntesis. ISBN: 9788477382218

Cebrián-Herreros, Mariano (2009). "Expansión de la ciberradio". Enl@ce Revista venezolana de información, tecnología y conocimiento, v. 6, n. 1, pp. 11-23.

http://produccioncientificaluz.org/index.php/enlace/ article/view/13474/13458

Contreras-Pulido, Paloma; Aguaded-Gómez, José-Ignacio (2012). "La voz de los presos en la radio: del objeto del discurso a constructores del mismo". Revista comunicación, v. 1, n. 10, pp. 1065-1075.

https://goo.gl/CF0a63

Contreras-Pulido, Paloma; González-Mairena, Miguel; Aguaded-Gómez, José-Ignacio (2014). “Programar una radio social en la universidad: el propósito Penélope de UniRadio". Edmetic, Revista de educación mediática y TIC, v. 3, n. 1, pp. 112-130.

http://helvia.uco.es/xmlui/handle/10396/11672

Díaz-Aledo, Loles (2007). “La representación de la discapacidad en los medios de comunicación. Cómo lograr una presencia más adecuada". Comunicación e cidadanía, n. 1, pp. 289-306. https://dialnet.unirioja.es/descarga/articulo/2542950.pdf 
Echeverría, Javier (2008). “Apropiación social de las tecnologías de la información y la comunicación". Revista CTS, v. 4, n. 10, pp. 171-182.

https://goo.gl/6EkbWJ

Escandell-Montiel, Daniel (2015). "Nanomedios: del blog aficionado a la información profesional en la Red". El profesional de la información, v. 24, n. 3, pp. 329-337.

https://doi.org/10.3145/epi.2015.may.13

Fernández-Sande, Manuel; Leal-Leal Carlos-Manuel (2015). "La radio como instrumento de rehabilitación y recuperación en trastorno mental severo y crítico: experiencias en la Comunidad de Madrid". En: Oliveira, Madalena; Ribeiro, Fabio (coords.). Radio, sound and internet. Braga (Portugal): CECS-Centro de Estudos de Comunicação e Sociedade, Universidade do Minho, pp. 99-110. ISBN: 9789898600370 http://www.lasics.uminho.pt/ojs/index.php/cecs_ebooks/ article/view/2169

Herrera-Damas, Susana (2003). "Rasgos diferenciales de la radio como medio de participación". Revista de comunicación, v. 1, pp. 25-40.

http://udep.edu.pe/comunicacion/rcom/pdf/2003/Art02540.pdf

INE (2008). Encuesta de discapacidad, autonomía personal y situaciones de dependencia (EDAD). Madrid: INE. http://www.ine.es/prensa/np524.pdf

Jiménez-Lara, Antonio (2009). "Nuevas tecnologías y discapacidad. Oportunidades y retos". En: Ledesma, Juan-Antonio (coord.). La imagen social de las personas con discapacidad. Estudios en homenaje a José Julián Barriga Bravo. Madrid: Ediciones Cinca, pp. 137-164. ISBN: 9788496889323 http://www.codajic.org/sites/www.codajic.org/files/ Laimagensocialdelaspersonascondiscapacidad_0.pdf

Kaplún, Mario (1998). Una pedagogía de la comunicación. Madrid: Ediciones de La Torre. ISBN: 9878479601858 http://perio.unlp.edu.ar/catedras/system/files/kaplun-el_ comunicador_popular_0.pdf

Katz, Elihu; Gurevitch, Michael; Haas, Hadassah (1973). "On the use of the mass media for important things". American sociological review, v. 38, n. 2, pp. 164-181.

https://goo.gl/zyJDFJ

https://doi.org/10.2307/2094393

Madrigal-Barrón, Patricia; Carrillo-Pascual, Elena (2014). "Visibilidad e integración. Una revisión sobre el papel de los medios audiovisuales en la construcción social de la discapacidad". En: Cairo, Heriberto; Finkel, Lucila (coords.). Actas del XI Congreso español de sociología 'Crisis y cambio: propuestas desde la sociología', 10-12 de julio de 2013, Madrid: Facultad de Ciencias Políticas y Sociología, Universidad Complutense de Madrid, pp. 1147-1158.

https://goo.gl/vx1Dw9

Mateo-Mateo, Abraham (2010). Una radio sin barreras. Radio y discapacidad. Análisis del tratamiento de la discapacidad en la radio. Programas por y para discapacitados y la utilidad de la radio como terapia ante la discapacidad. Proyecto fin de master. Madrid: Universidad Rey Juan Carlos.
McQuail, Denis; Blumler, Jay; Brown, Joseph (1972). "The television audience: a revised perspective". En: McQuail, Denis (ed.). Sociology of mass communications. Harmondsworth: Penguin Books. ISBN: 9780140809619

Merayo-Pérez, Arturo (2000). Para entender la radio. Salamanca: Publicaciones Universidad Pontificia de Salamanca. ISBN: 9788472992849

Mertens, Donna (2015). Research and evaluation in Education and Psychology: Integrating diversity with quantitative, qualitative and mixed methods. Thousand Oaks: Sage. ISBN: 9781412971904

Muñoz-González, José-Javier; Gil, César (1994). La radio. Teoría y práctica. Madrid: IORTV. ISBN: 9788486984939

OMS (2001). Clasificación internacional del funcionamiento, de la discapacidad y de la salud (CIF). Madrid: Imserso, Ministerio de Asuntos Sociales. ISBN: 8484460347

ONU (2008). Convención sobre los derechos de las personas con discapacidad.

http://www.un.org/esa/socdev/enable/documents/tccconvs. $p d f$

Ortiz, Miguel-Ángel; Volpini, Federico (1995). Diseño de programas de radio: guiones, géneros y fórmulas. Barcelona: Paidós. ISBN: 9788449301438

Perujo-Serrano Francisco (2002). "Discapacidad y medios de comunicación; entre la información y el estereotipo". Ámbitos, n. 7-8, pp. 249-277.

http://www.redalyc.org/articulo.oa $?$ id $=16800814$

Rappaport, Julian (1987). "Terms of empowerment/exemplars of prevention: Toward a theory for community psychology". American journal of community psychology, v. 15, n. 2, pp. 121-148.

http://www.new-gallery-of-art.com/pdf/antioch/6.\%20 Rappaport\%20(1987).pdf

https://doi.org/10.1007/BF00919275

Rodero, Enma (2008). "Educar a través de la radio". Signo y pensamiento, n. 52, pp. 97-109.

http://www.redalyc.org/articulo.oa? ?id $=86005207$

Ruiz-Olabuénaga, José-Ignacio (2012). Metodología de la investigación cualitativa. Bilbao: Universidad de Deusto. ISBN: 9788498303360

Sabés-Turmo, Fernando; Parra-Valcarce, David (2014). “Del consumidor al prosumidor: responsabilidades legales de los informers". El profesional de la información, v. 23, n. 5, pp. 511-518.

http://dx.doi.org/10.3145/epi.2014.sep.08

Sánchez-de-Amo, Antonio (2003). "Los medios de comunicación ante la discapacidad". Acciones e investigaciones sociales, n. 17, pp. 183-214.

https://papiro.unizar.es/ojs/index.php/ais/article/ view $/ 257 / 251$

Shalock, Robert et al. (2011). Discapacidad intelectual. Definición, clasificación y sistemas de apoyo. 11a ed. Madrid: Alianza. ISBN: 9788420652627 


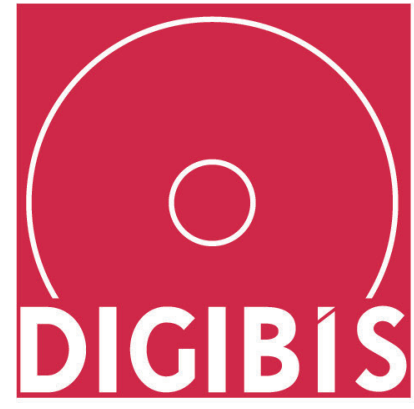

Digitalización enriquecida de fondo antiguo y patrimonial

Software de gestión para Bibliotecas, Archivos y Museos

DIGIBIB $^{\circ}$ DIGIARCH $^{\circ}$ DIGIMUS ${ }^{\circ}$

Recolector OAI-PMH DIGIHUB' de metadatos de diversos proveedores

DIGIBÍS transforma y enriquece las bibliotecas con aplicaciones de gestión digital de nueva generación.

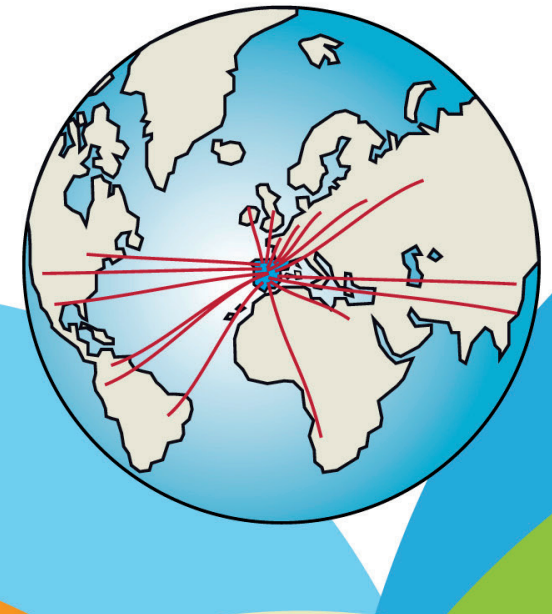

El usuario accede fácilmente a los recursos de la biblioteca desde cualquier lugar, en cualquier momento, con cualquier dispositivo.

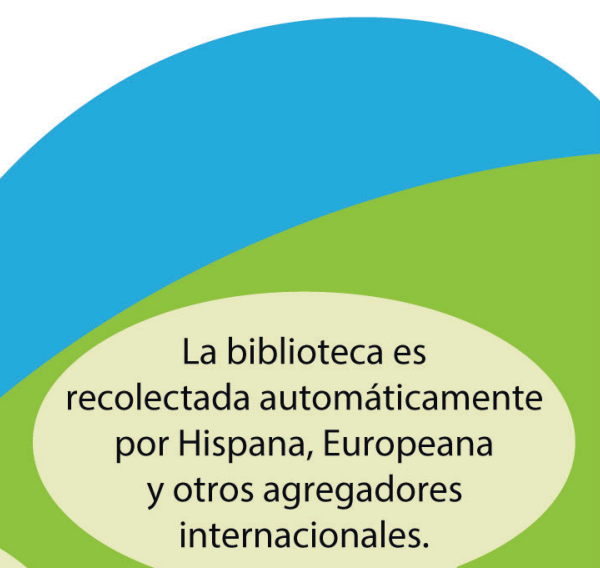

internacionales.
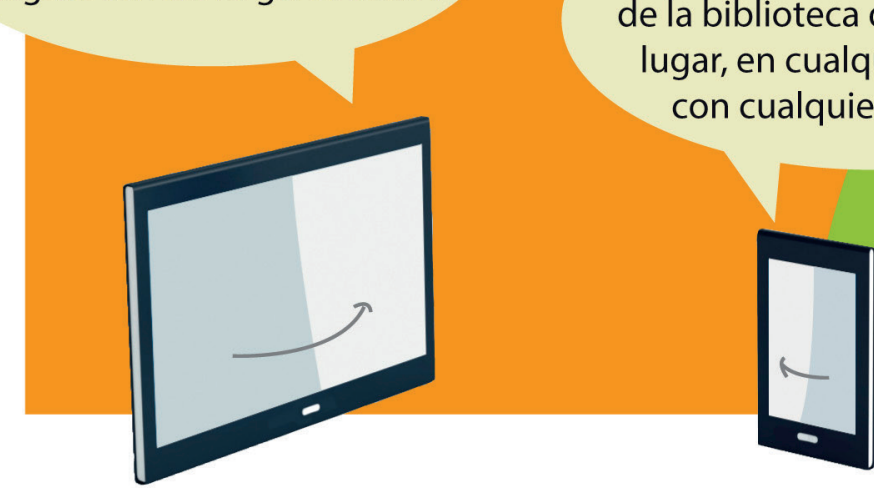